\title{
The Advanced Video Guidance Sensor: Orbital Express and the Next Generation
}

\author{
Richard T. Howard ${ }^{1 \mathrm{a}}$, Andrew F. Heaton ${ }^{2}$, Robin M. Pinson ${ }^{2}$, Connie L. \\ Carrington ${ }^{1}$, James E. Lee ${ }^{3}$, Thomas C. Bryan ${ }^{1}$, Bryan A. Robertson ${ }^{4}$, Susan H. \\ Spencer ${ }^{3}$, and Jimmie E. Johnson ${ }^{3}$ \\ ${ }^{1}$ Avionics Systems Test Branch (EV21) \\ ${ }^{2}$ Guidance, Navigation, and Mission Analysis Branch (EV42) \\ ${ }^{3}$ Exploration Advanced Capabilities Office (VP33) \\ ${ }^{4}$ Flight and Ground Computers Branch (EI31) \\ NASA Marshall Space Flight Center, Huntsville, AL 35812, USA \\ 1a 256-544-3536, ricky.howard@nasa.gov
}

\begin{abstract}
The Orbital Express (OE) mission performed the first autonomous rendezvous and docking in the history of the United States on May 5-6, 2007 with the Advanced Video Guidance Sensor (AVGS) acting as one of the primary docking sensors. Since that event, the OE spacecraft performed four more rendezvous and docking maneuvers, each time using the AVGS as one of the docking sensors. The Marshall Space Flight Center's (MSFC's) AVGS is a nearfield proximity operations sensor that was integrated into the Autonomous Rendezvous and Capture Sensor System (ARCSS) on OE. The ARCSS provided the relative state knowledge to allow the OE spacecraft to rendezvous and dock. The AVGS is a mature sensor technology designed to support Automated Rendezvous and Docking (AR\&D) operations. It is a video-based laser-illuminated sensor that can determine the relative position and attitude between itself and its target. Due to parts obsolescence, the AVGS that was flown on OE can no longer be manufactured. MSFC has been working on the next generation of AVGS for application to future Constellation missions. This paper provides an overview of the performance of the AVGS on Orbital Express and discusses the work on the Next Generation AVGS (NGAVGS).
\end{abstract}

Keywords: Sensors, docking, proximity operations, relative navigation, rendezvous, video PACS: 07.07.Df

\section{INTRODUCTION}

The Orbital Express (OE) mission consisted of a pair of spacecraft outfitted with all of the hardware and software necessary to demonstrate the technical feasibility of different operations necessary for performing satellite servicing. The different operations performed during the $\mathrm{OE}$ mission were all completely automated and consisted of spacecraft rendezvous, spacecraft proximity operations, spacecraft docking, spacecraft free-flyer capture, fluid transfers, and Orbital Replacement Unit (ORU) transfers. The mission was primarily funded by the Defense Advanced Research Projects Agency (DARPA), with additional funding provided by Boeing and NASA. NASA supplied the flight software, ground testing, and some hardware and firmware support for the Advanced Video Guidance Sensor (AVGS). In addition, NASA tested the entire OE relative navigation sensor system in open-loop fashion. The two spacecraft, the Boeing-built Autonomous Space Transport Robotic Operations (ASTRO) and the Ball Aerospace built Next Generation Serviceable Satellite (NEXTSat) were launched in a mated configuration on an Atlas V launch vehicle on March 8, 2007. Following deployment of the OE payload, initial mission operations consisted of several fluid transfer and ORU transfer operations that were conducted in the mated configuration. The spacecraft were also maneuvered relative to one another using the robotic arm on the ASTRO to check out the 
relative navigation sensor system. Beginning on May 5, 2007, the spacecraft were allowed to separate relative to one another and undergo a series of automated rendezvous and docking missions with different characteristics and varying maximum separation ranges. In between rendezvous and docking maneuvers, additional fluid and ORU transfers occurred.

One of the key technologies required for satellite servicing is Automated Rendezvous and Docking (AR\&D). AR\&D was demonstrated several times during the mission, including the first autonomous docking in the history of the United States space program on May 5-6, 2007. The ASTRO spacecraft had the Autonomous Rendezvous and Capture Sensor System (ARCSS) suite of sensors on board. The ARCSS sensors consisted of a set of two visible light cameras, an infrared camera, a laser rangefinder, and the OE AVGS. All of the camera data was fed into a computer that processed the camera images using Boeing-developed algorithms, and then the computer combined that output with the information from the AVGS and the laser rangefinder onto both the data bus for the primary mission computer and onto a solid-state recorder for later transmission to the ground.

\section{ORBITAL EXPRESS ADVANCED VIDEO GUIDANCE SENSOR}

The AVGS as built for the OE mission was specified and designed to guide a spacecraft in to dock with another spacecraft. The AVGS consists of two sets of laser diodes at wavelengths of 800 and 850 nanometers, a mirror through which the lasers fire, a camera that images the return from the lasers, and hardware, software, and firmware that process the returned images into relative position and attitude data. The sensor is designed to interact with a retro-reflective target. The target has filters that allow one wavelength of AVGS laser to pass through and be reflected while blocking the other wavelength. The target retro-reflectors are arranged in a pattern known to the AVGS software. The sensor fires one set of lasers and captures an image, then it fires the second set of lasers and captures a second image. When this second image is subtracted from the first image and an intensity threshold is used, virtually all of the background clutter is eliminated. The remaining data is converted into a set of spots, and the spots are compared to the target pattern. Once a set of spots matching the target is found, the software computes the relative position and attitude between the target and the sensor. This data is output from the sensor and fed into the ARCSS computer for use by the Guidance and Relative Navigation (GRN) algorithms, as well as for archiving. There are several modes of operation in the AVGS. The primary AVGS modes of operation used during the OE mission were the following. 1) Standby (in which the sensor sends out status messages while awaiting further commands), 2) Acquisition (in which the sensor is actively seeking a target), and 3) Tracking (the sensor is actively tracking a target). More information about AVGS can be found in other publications (Howard et al, 2004; LeCroy, Hallmark, and Howard, 2007).

\section{Advanced Video Guidance Sensor Specifications}

The accuracy specifications for the OE AVGS are shown in Table 1. The specifications become more stringent as range decreases because spacecraft docking occurs at close ranges, where the best accuracy is required. Since the AVGS uses an imager chip and a fixed-focus lens, the accuracy of the AVGS gets better as the range decreases. There are two specifications from 10 to 30 meters, since the AVGS OE target has both a Long Range Target (LRT) and Short Range Target (SRT) that are tracked over different ranges of operation.

TABLE 1. Orbital Express AVGS Accuracy Requirements

\begin{tabular}{|c|c|c|c|c|}
\hline $\begin{array}{c}\text { Operating Range } \\
(\mathrm{m})\end{array}$ & $\begin{array}{c}\text { Range } \\
(\mathrm{mm})\end{array}$ & $\begin{array}{c}\text { Azimuth, Elevation } \\
\text { (Degrees) }\end{array}$ & $\begin{array}{c}\text { Roll } \\
\text { (Degrees) }\end{array}$ & $\begin{array}{c}\text { Pitch/Yaw } \\
\text { (Degrees) }\end{array}$ \\
\hline $1-3$ & \pm 12 & \pm 0.033 & \pm 0.13 & \pm 0.2 \\
\hline$>3-5$ & \pm 35 & \pm 0.033 & \pm 0.25 & \pm 0.33 \\
\hline$>5-10$ & \pm 150 & \pm 0.035 & \pm 0.45 & \pm 0.7 \\
\hline$>10-30(\mathrm{SR})$ & \pm 1500 & \pm 0.037 & \pm 1.3 & \pm 2 \\
\hline$>10-30(\mathrm{LR})$ & \pm 150 & \pm 0.027 & \pm 0.15 & \pm 0.7 \\
\hline$>30-50$ & \pm 400 & \pm 0.03 & \pm 0.25 & \pm 1.2 \\
\hline$>50-100$ & \pm 1666 & \pm 0.033 & \pm 0.5 & \pm 2.4 \\
\hline$>100-300$ & $\pm 15,000$ & \pm 0.035 & \pm 1.4 & \pm 7.0 \\
\hline
\end{tabular}


The overlap region for tracking the two targets simultaneously is nominally from 10 meters to 30 meters. The data output rate of the OE AVGS was $5 \mathrm{~Hz}$, while the sensor internally tracked the target at $10 \mathrm{~Hz}$. The sensor had a field-of-view (FOV) of $+/-8$ degrees, and was required to track the target while it was within a 7 degree cone about the center of the FOV.

\section{Orbital Express AVGS Performance}

A number of different scenarios and operations were carried out with the AVGS powered on. All operations contained a segment during which the AVGS was in a static, fixed position relative to the SRT, and the majority of the scenarios involved dynamic motion of the target relative to the sensor. Some dynamic motion occurred while the two spacecraft were attached by a robotic arm, but most dynamic motion occurred during the unmated scenarios while the two spacecraft were free-flying relative to one another. During the Orbital Express mission, the AVGS performed extremely well. Fig. 1 is a picture of the NEXTSat spacecraft taken by one of the ARCSS visible light cameras. The four AVGS LRT retro-reflectors are clearly visible at the bottom left, bottom right, top right, and near the top left of the ASTRO body. The AVGS SRT (also containing four closely-spaced retro-reflectors) is barely visible in gray at the bottom of the picture.

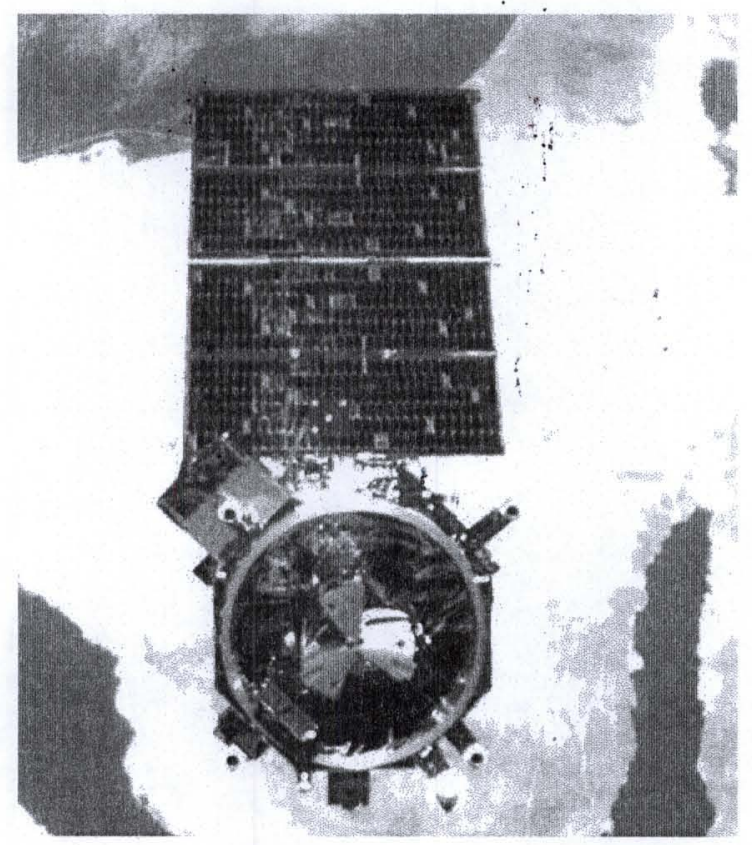

FIGURE 1. The NEXTSat Spacecraft in Orbit (DARPA, 2007).

The AVGS played a key role in the unmated scenarios of the OE mission. AVGS was active for all six of the unmated operations, three of which ended in docking and two in free-flier capture. During the sixth unmated operation, the two spacecraft were sent to different orbits for End of Life. The AVGS also collected data during ARCSS sensor checkout, berthing after separation ring ejection, the two mated calibration procedures (AT-UTIL25), and a 5-hour sensor stress test. The AVGS contributed significantly to the successful dockings and free-flier captures during all OE unmated operations. The AVGS tracked continuously while the range was less than 60 meters and while on the approach corridor during all proximity operations and docking.

When the AVGS was powered on it was primarily in Track Mode or Acquisition Mode. During the AVGS warmup the sensor was in Standby Mode. Throughout the mission the Diagnostic, Maintenance, and Reset Modes were occasionally used. These modes operated successfully each time they were called. While AVGS was in Track Mode the output data rate was consistently $5 \mathrm{~Hz}$. Twice during the mission a new ILOAD developed on the ground was sent to the AVGS and implemented. This process was run with ease and efficiency both times. The AVGS spent over 56.8 hours in Track Mode or Acquisition Mode while on orbit. 
The region where the AVGS was able to track its target was a critical element in the performance analysis, including the maximum and minimum ranges for which the Short Range Target (SRT) and the Long Range Target (LRT) were able to be successfully tracked. The SRT was required to be tracked from mated range out to 30 meters. On departure the SRT steadily tracked to $32.2-34.2$ meters, which is beyond the requirement. During approach the SRT consistently began tracking between 31.8 - 32.3 meters. The minimum range requirement for LRT tracking was 10 meters. On departure the AVGS tracked the LRT starting at approximately $8.7-8.9$ meters, while during approach the LRT was tracked until $8.7-9.0$ meters. Inside the critical proximity operations range of 60 meters, while the target was in the approach corridor, the AVGS solidly tracked its targets, greatly contributing to each successful docking. The AVGS typically tracked the LRT until $100-110$ meters when the LRT was in the FOV. Most scenarios were not designed to keep the AVGS in the targets' FOV past this range. Throughout the mission, the AVGS remained in track until a few minutes prior to leaving the departure corridor, or began tracking within a few minutes of entering the approach corridor. Due to anomalies early in Scenario 3-1, the AVGS target was pointed at the AVGS earlier than planned during remating, and the AVGS began tracking the LRT at 150 meters on the approach. The AVGS worked well out to 150 meters, even though pre-flight testing took place at a maximum range of 100 meters.

The AVGS successfully tracked a large range of attitudes, azimuths and elevations. The mission was not designed to test the AVGS tracking envelope other than during the ARCSS Check-out. The data available for analysis encompasses a range of pitch values from -26 through 26 degrees, yaw from -23 through 10 degrees, azimuth from 7 through 7 degrees and elevation from -6 to 8 degrees. The maximum tilt angle on three scenarios exceeded the 25 degree requirement at ranges greater than 60 meters.

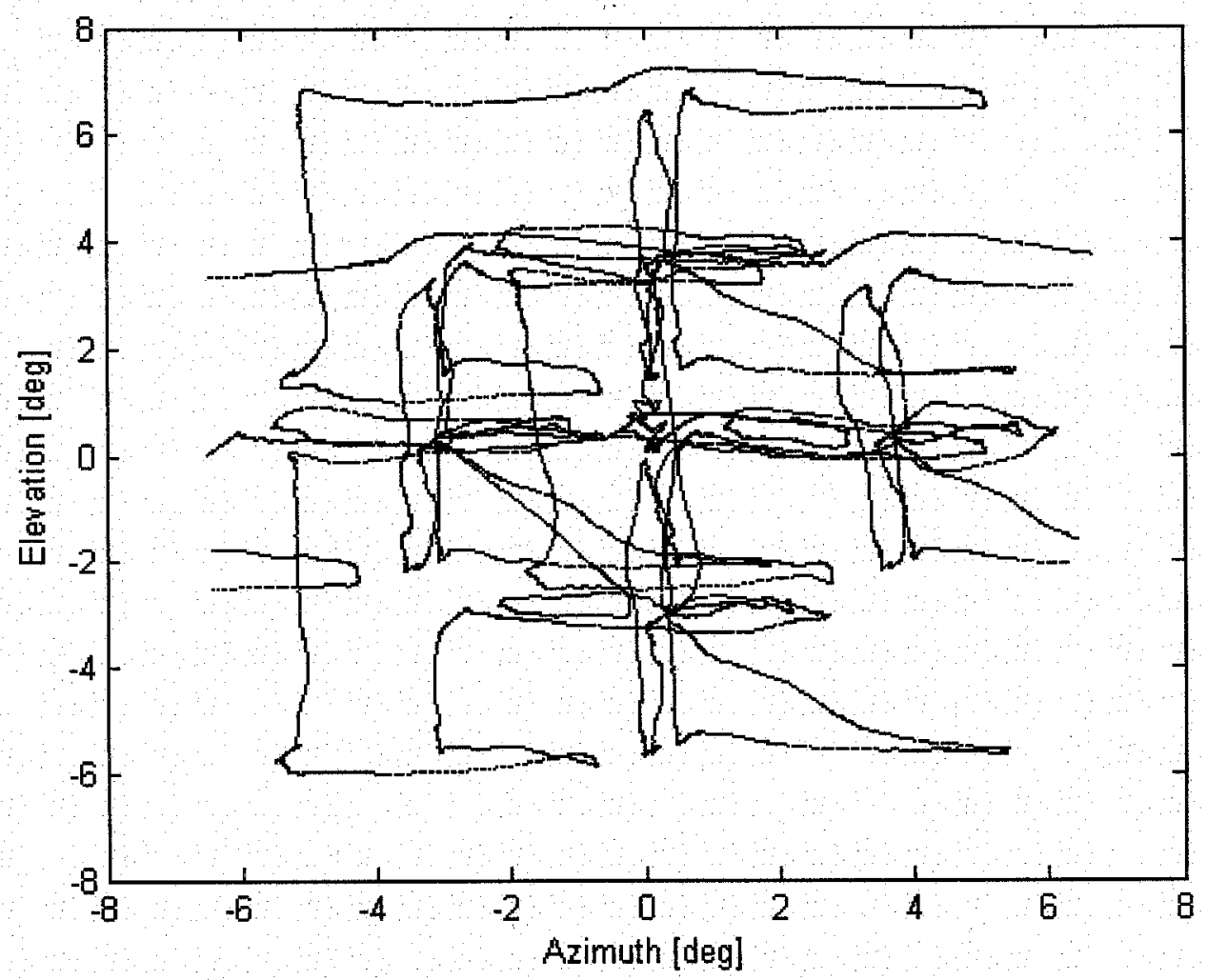

FIGURE 2. AVGS Azimuth and Elevation plot during ARCSS checkout.

Scenario 1-1A ARCSS Check-out

Scenario 1-1A consisted of the Robotic Arm maneuvering the NEXTSat spacecraft into a variety of positions and attitudes relative to the ASTRO spacecraft through two scripts. Excursions included points within the operating 
corridor and beyond that edge. Two different automated arm motion scripts were run; one examined sensor performance at the expected edges of operation. The other script evaluated what happens when a sensor loses and regains track of its target at different positions and attitudes. Each script was repeated one time. The ARCSS checkout was designed to test the operational limits of the other ARCSS sensors, but it benefited the AVGS as well.

In this operation, the AVGS performed very well, effectively tracking beyond its 10 degree tilt limit specifications at ranges closer than $30 \mathrm{~m}$. The AVGS repeatedly reached a ten degree tilt angle, and successfully tracked at a tilt angle more than double the requirement. In addition to the tilt angle, the pitch and yaw were both tracked close to ten degrees individually. Fig. 2 shows Azimuth vs. Elevation for one of the ARCSS checkout scripts. The motion went to the edges of the sensor's operation and a little bit beyond in Azimuth. Positions of the SRT in the FOV (azimuth and elevation) were compared between the two executions of the same script. The match was excellent, with slight variations that can be attributed to robotic arm vibration and noise, demonstrating AVGS repeatability.

\section{AVGS Mated Data}

There were fifteen distinct times when the AVGS was in Track Mode while the two vehicles were in the mated configuration. Three of these periods were taken during operations when the vehicles were not scheduled to perform unmated operations. These three opportunities were the longest sets of mated static data available, including one 5.2 hour sample. The remainder of the mated data was taken immediately before the vehicles undocked, or while the sensor collected data after the two vehicles were rigidly docked. The mated data is crucial to assessing the performance of the AVGS at close ranges; the docking mechanism used for OE had a very tight tolerance that essentially was below the threshold of the AVGS to detect, and made an excellent source of "truth data."
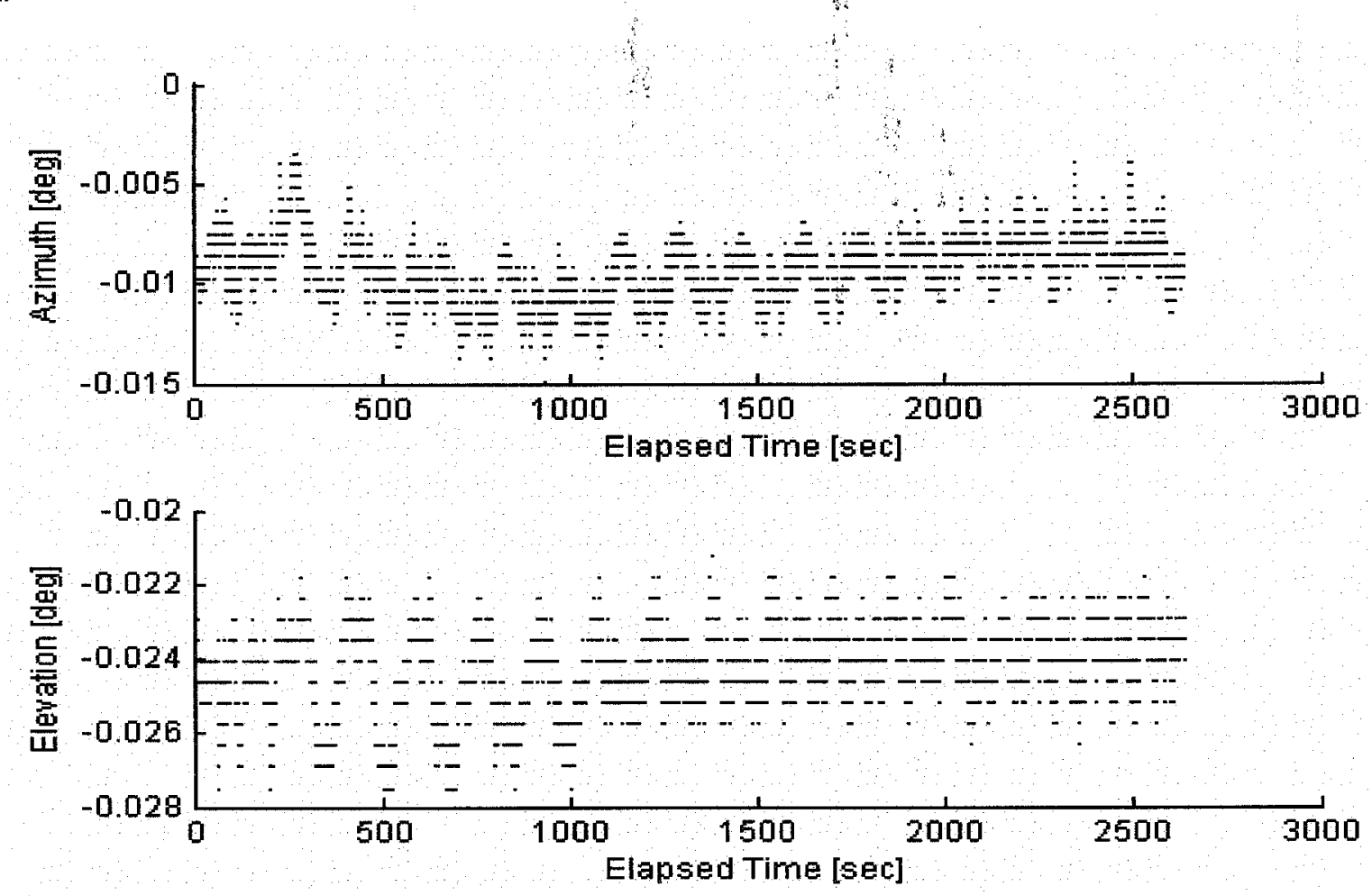

FIGURE 3. AVGS Static Azimuth and Elevation Data.

Statistics were developed for the fifteen mated data samples. These statistics represent the repeatability of the AVGS and the repeatability of the docking mechanism. The mated data standard deviation was an order of magnitude smaller than the specifications, and the mean and median also fell within 1 sigma. When docked, the specifications encompass the bias from the desired zero solution as well as noise from the sensor. Biases can be taken out with ILOAD updates, but the biases were not deemed large enough to remove by the program management team. Fig. 3 is two plots, Azimuth vs. time and Elevation vs. time, for one of the mated data sets. The 
data does not vary much about the mean, and the resolution of the sensor data, 0.000573 degrees, is visible as the discrete levels in the plots.

For all fifteen cases, the mean range varied less than a millimeter from the 1.220 meter zero point, with a standard deviation of less than $0.75 \mathrm{~mm}$, compared to the specification of $12 \mathrm{~mm}$. For azimuth, the largest mean added to the largest standard deviation is less than $60 \%$ of the 0.033 deg spec. The elevation bias was close to the 1 sigma specification of 0.033 degrees, though it did not vary by more than 0.013 deg between the fifteen cases. The largest elevation standard deviation was less than $15 \%$ of the spec. The largest standard deviation for pitch, added to the largest mean, was approximately $60 \%$ of the $0.2 \mathrm{deg}$ specification. This encompasses the bias and its noise. Yaw was $80 \%$ of the $0.2 \mathrm{deg}$ specification for the same combination. The biases could have been corrected through an ILOAD, but that correction was not considered necessary for mission operations. The roll bias varied by 0.09 degrees, which is under the specification of $0.15 \mathrm{deg}$. The largest roll standard deviation was under $20 \%$ of the specification. Overall, very few data points fell outside the 1 sigma boundaries while in the mated configuration, well within the required 3 sigma specification. The mated data showed high repeatability in the AVGS performance.

\section{Dual Track}

Between the ranges of 9 meters and 32 meters, the AVGS tracked both the LRT and SRT simultaneously. This region is commonly referred to as Dual Track. During Dual Track the AVGS is measuring the relative position and attitude of NEXTSat through both the SRT and LRT. At the same point in time, the two solutions should be the same, since the spacecraft cannot physically have two attitudes and positions.

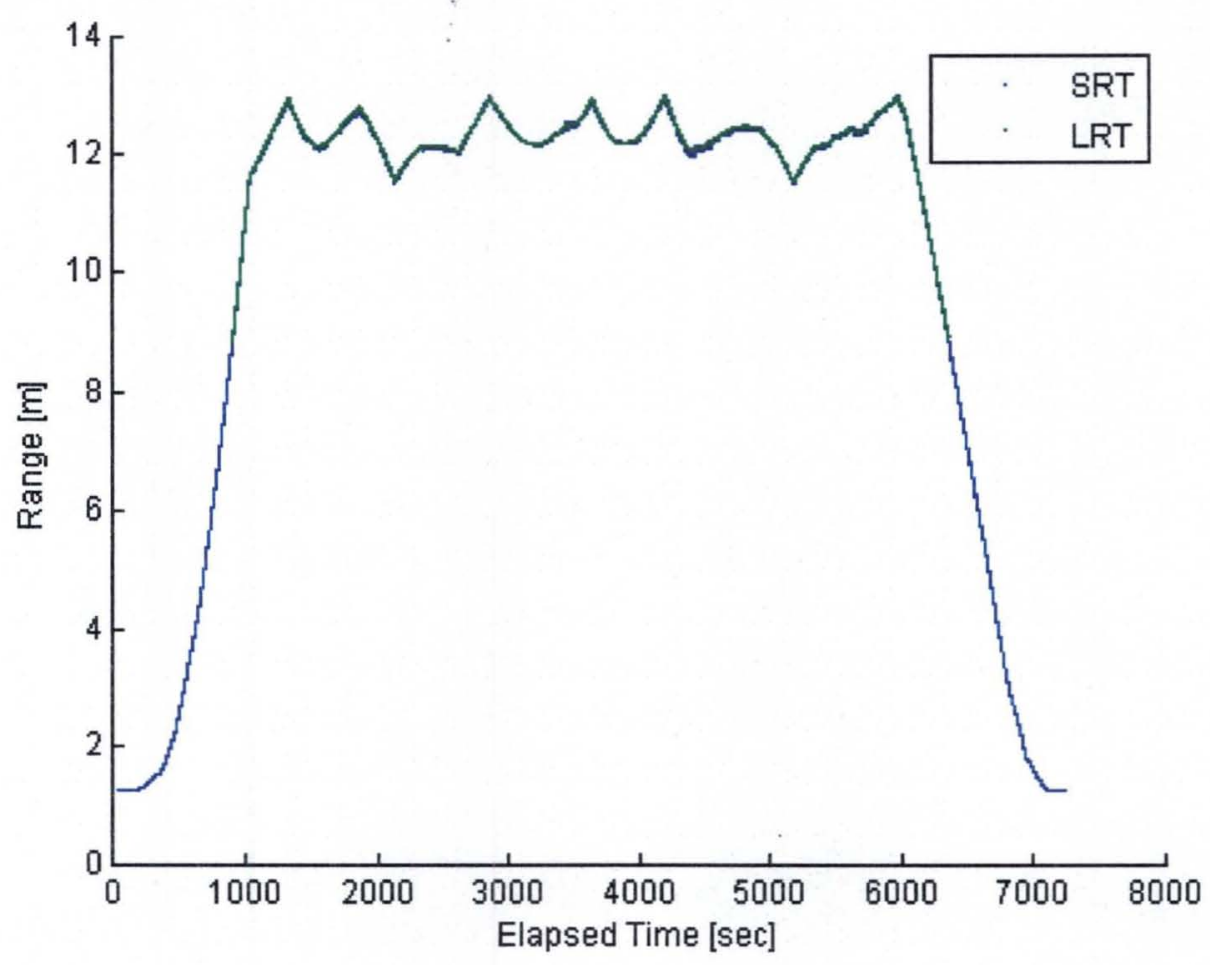

FIGURE 4. AVGS range to both targets during Scenario 2-1.

The AVGS solutions from the LRT and SRT were closely correlated and remained consistent throughout the scenarios. The SRT solution was subtracted from the LRT solution during the dual track regions with statistics calculated on the solution difference. Due to the large range that Dual Track covered in addition to two different targets, four requirements specifications cover the Dual Track range. The tightest specification, which was the LRT between $10-30$ meters, was used for comparison. The range noise, represented by the standard deviation, was less 
than half of the specification of $+/-0.150 \mathrm{~m}$. The largest noise for azimuth and elevation was slightly over half of the $+/-0.027 \mathrm{deg}$ specification. Pitch and yaw noise were also under half of their specification of $+/-0.7 \mathrm{deg}$, with the largest roll noise being close to $80 \%$ of the $+/-0.15$ deg specification.

The plot in Fig. 4 shows the overall motion of the sensor relative to the target along the $\mathrm{X}$-axis (which was essentially the range) during the entirety of Scenario 2-1. The two spacecraft separated to a distance of a little more than 10 meters, held position there for almost two hours, and then approached and docked. The two different targets were tracked simultaneously while the range exceeded approximately 8.8 meters, and the data from the two targets is nearly indistinguishable.

\section{NEXT GENERATION ADVANCED VIDEO GUIDANCE SENSOR (NGAVGS)}

Video-based sensors have been under regular development, testing, and upgrading at MSFC for almost 20 years. The first sensor flown was the Video Guidance Sensor (VGS) in 1998, followed by the AVGS on the Demonstration of Autonomous Rendezvous Technologies (DART) mission (Howard and Bryan, 2007). Based on lessons learned and due to the fact that some of the components of the OE AVGS are no longer available, MSFC has been working on the next generation of the AVGS. Since this work is being performed in order to support future Constellation missions, radiation is a bigger concern than it was on OE. The baseline NGAVGS performance requirements are similar to the OE AVGS requirements with regard to range of operation and accuracy, but it must be noted that the sensor performance depends heavily on the target configuration used. The NGAVGS, like its predecessors, looks at the spots of light generated by illuminating a retro-reflective target. The accuracy at which the sensor tracks those spots is a function of reflector size, range, and position in the FOV.

The weight, volume, and power consumption estimates for the NGAVGS are expected to be on the order of the AVGS values or slightly better. The AVGS weighed $20 \mathrm{lbs}$, was approximately $7 \times 10 \times 12$ inches, and consumed approximately 14 Watts in Standby mode and 35 Watts in Tracking mode (the most power intensive mode of operation).

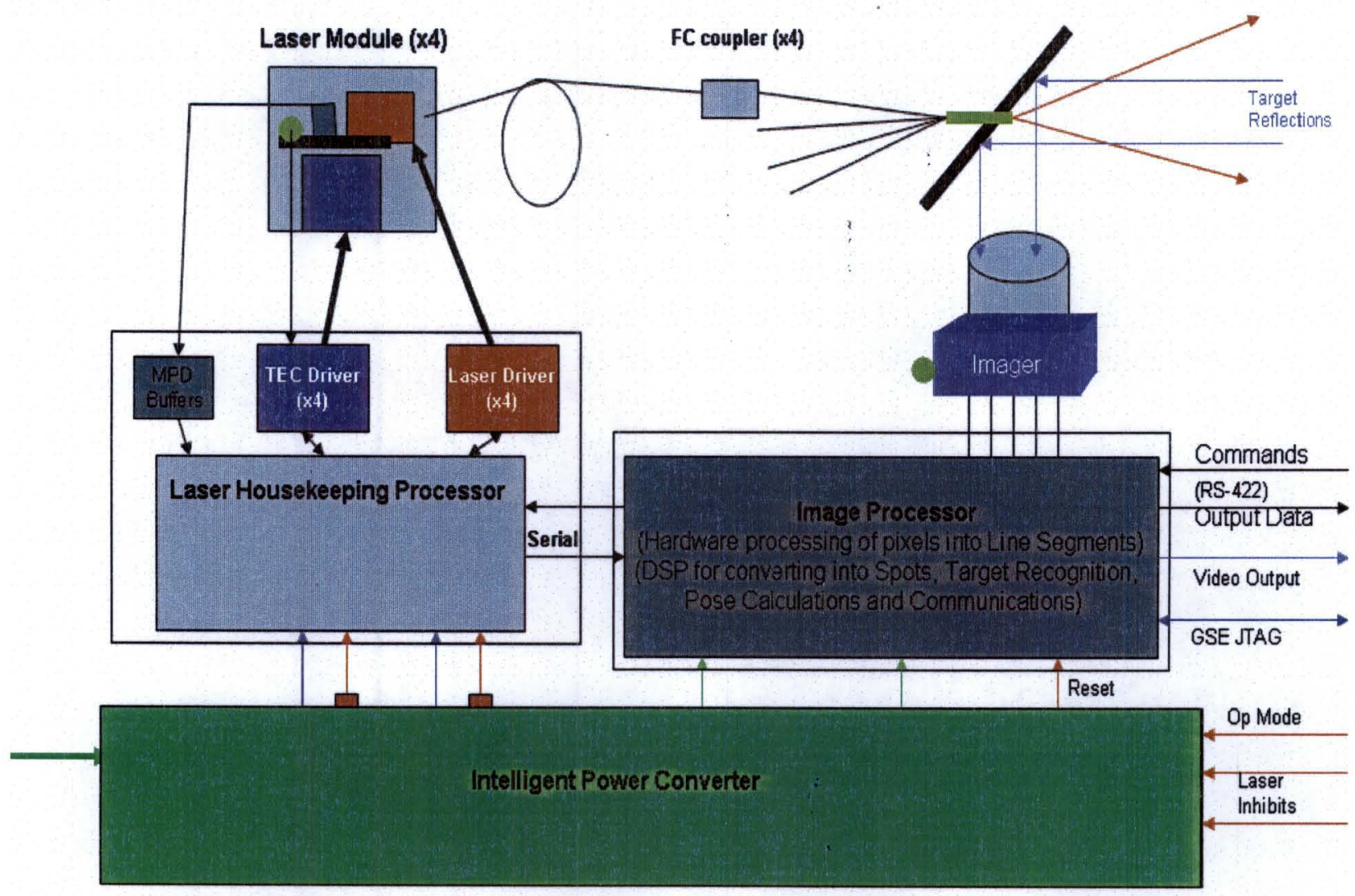

FIGURE 5. Block Diagram Showing Some of the Connections and Functionality of the NGAVGS. 
A general block-diagram of the NGAVGS is shown in Fig. 5. The NGAVGS functions in similar fashion to the AVGS - it takes two sequential pictures illuminated by different wavelengths of laser light, subtracts one picture from the other, creates spots from the image data, matches spots to the known target geometry, and computes the relative position vector and relative attitude information.

During Acquisition or Tracking, image acquisition is initiated by the digital signal processor (DSP). The DSP generates a command to the Field Programmable Gate Array (FPGA) to begin a cycle. The FPGA starts by issuing a fire laser command to the Laser Housekeeping Processor. The lasers are fired and the imager accumulates charge for a predetermined amount of time (the integration time or exposure time). Once the integration time is complete, the image is passed from the imager to the FPGA where it is stored in external memory. Two complete images are accumulated in this manner with each image being stored in separate memory. The differences in the two images are primarily caused by the different wavelengths of lasers that were fired during the integration time. The two images are then compared by the FPGA, and the comparison data is compressed and passed to the DSP where pattern matching is performed. A final solution is calculated and transmitted out thru the RS- 422 interface. The image can be seen in real time thru the Video Output port.

A breadboard version of the NGAVGS was built at MSFC to test the new components that were chosen to replace the obsolete components. Once the breadboard had been successfully tested, a brassboard version of the NGAVGS was built. The brassboard has been tested at ranges from 1 to 15 meters and at 300 meters, to verify that the design approach was sound. Future work includes plans to test the unit at ranges from 1 to 300 meters continuously, as well as using outdoor ranges to perform longer range testing.

\section{CONCLUSION}

The AVGS played a vital role in supporting the extremely successful Orbital Express mission. The sensor performed significantly better than required in a number of categories, including extending the SRT track range and outstanding repeatability between different mated operations. The solutions from the SRT and the LRT during Dual Track had excellent correlation. The amount of data collected, both static and dynamic, was substantial, providing the basis for more analysis and a better understanding of on-orbit AR\&D. Future generations of video-based sensors will benefit from the experiences gained on OE. The NGAVGS is one such sensor, and it is following in the footsteps of its successful progenitors, the AVGS and the VGS.

\section{REFERENCES}

Defense Advanced Research Projects Agency (DARPA), "Orbital Express On-Orbit Mission Updates" (2007), http://www.darpa.mil/orbitalexpress/mission updates.html, accessed October 2, 2007.

Howard, R. T., and Bryan, T. C., "DART AVGS Flight Results", in Sensors and Systems for Space Applications, edited by R. T. Howard and R. D. Richards, Proceedings of SPIE Vol. 6555, 65550L, Bellingham, WA, 2007, pp. 1-10.

Howard, R. T., Johnston, A. S., Bryan, T. C., and Book, M. L., "Advanced Video Guidance Sensor (AVGS) development testing", in Spaceborne Sensors, edited by R. D. Habbit, Jr. and P. Tchoryk, Jr., Proceedings of SPIE Vol. 5418, Bellingham, WA, 2004, pp. 50-60.

LeCroy, J. E., Hallmark, D. S., and Howard, R. T., "Effects of Optical Artifacts in a Laser-Based Spacecraft Navigation Sensor", in Sensors and Systems for Space Applications, edited by R. T. Howard and R. D. Richards, Proceedings of SPIE Vol. 6555,655507 , Bellingham, WA, 2007, pp. 1-11. 